\title{
Dietary acrylamide and cancer of the large bowel, kidney, and bladder: Absence of an association in a population-based study in
} Sweden

\author{
LA Mucci*, ${ }^{, 1,2}$, PW Dickman ${ }^{1,3}$, G Steineck ${ }^{3}$, H-O Adami ${ }^{1,2}$ and K Augustsson' \\ 'Department of Medical Epidemiology, Karolinska Institutet, Box 281, SE 17177 Stockholm, Sweden; ${ }^{2}$ Department of Epidemiology, Harvard School of \\ Public Health, 677 Huntington Avenue, 9th floor, Boston, MA 021 15, USA; ${ }^{3}$ Department of Oncology and Pathology, Clinical Cancer Epidemiology, \\ Karolinska Institutet, SE 17176 Stockholm, Sweden
}

\begin{abstract}
Recently, disturbingly high levels of acrylamide were unexpectedly detected in widely consumed food items, notably French fries, potato crisps, and bread. Much international public concern arose since acrylamide has been classified as a probable carcinogen, although based chiefly on laboratory evidence; informative human data are largely lacking. We reanalysed a population-based Swedish case-control study encompassing cases with cancer of the large bowel $(N=59$ I), bladder $(N=263)$ and kidney $(N=133)$, and 538 healthy controls, assessing dietary acrylamide by linking extensive food frequency data with acrylamide levels in certain food items recorded by the Swedish National Food Administration. Unconditional logistic regression was used to estimate odds ratios, adjusting for potential confounders. We found consistently a lack of an excess risk, or any convincing trend, of cancer of the bowel, bladder, or kidney in high consumers of 14 different food items with a high (range $300-1200 \mu \mathrm{g} \mathrm{kg}^{-1}$ ) or moderate (range $30-299 \mu \mathrm{g} \mathrm{kg}{ }^{-1}$ ) acrylamide content. Likewise, when we analysed quartiles of known dietary acrylamide intake, no association was found with cancer of the bladder or kidney. Unexpectedly, an inverse trend was found for large bowel cancer ( $P$ for trend $0.0 \mathrm{I}$ ) with a $40 \%$ reduced risk in the highest compared to lowest quartile. We found reassuring evidence that dietary exposure to acrylamide in amounts typically ingested by Swedish adults in certain foods has no measurable impact on risk of three major types of cancer. It should be noted, however, that relation of risk to the acrylamide content of all foods could not be studied.

British Journal of Cancer (2003) 88, 84-89. doi:I0.1038/sj.bjc.6600726 www.bjcancer.com
\end{abstract}

(c) 2003 Cancer Research UK

Keywords: acrylamide; kidney cancer; large bowel cancer; bladder cancer; diet

Acrylamide is classified by the International Agency for Research on Cancer (IARC) as a probable human carcinogen (IARC, 1994). In April 2002, a Swedish food survey created international public health alarm by its report of substantially elevated levels of acrylamide in frequently consumed food products (Swedish National Food Administration (NFA), 2002) and in particular, in potato crisps, French fries, and crisp bread. Acrylamide formation probably occurs as a result of a reaction between amino acids and reducing sugars during the heating of starch-rich foods to high temperatures (Mottram et al, 2002; Stadler et al, 2002).

Although animal models support a dose-response relation between acrylamide and cancer at multiple sites (Bull et al, 1984; Johnson et al, 1986), no epidemiological study to date has examined whether higher intake of foods containing high acrylamide level increases the risk of any cancer. The Swedish NFA findings therefore led to much public concern in Europe and the United States. In response, we have analysed data from a

*Correspondence: Dr LA Mucci, Department of Epidemiology, Harvard School of Public Health, 677 Huntington Avenue, 9th floor, Boston, MA 02115 USA; E-mail: Imucci@hsph.harvard.edu

Received 23 September 2002; revised 17 October 2002; accepted 25 October 2002 population-based case-control study in Sweden to investigate whether higher intake of certain food items with higher acrylamide content increases the risk of cancers of the large bowel, bladder, or kidney.

\section{MATERIALS AND METHODS}

\section{Study population}

This investigation was undertaken in an existing population-based case-control study of a possible relation between heterocyclic amines in fried foods, and cancers of the large bowel and urinary tract (Augustsson et al, 1999). The study base comprised individuals born in Sweden between 1918 and 1942, and residing in Stockholm for at least 1 month between November 1992 and December 1994. Incident cases were identified in the Stockholm area from the population-based cancer registry and included the following sites: large bowel (ICD9 153 and 154), bladder (ICD9 188 ), and kidney (ICD9 189, excluding renal pelvis 189.1 and 189.3). Controls were randomly selected from the Register of Total Population during the study period, and frequency matched by age and gender. 
Survey questionnaires were mailed to 692 controls, and 875, 391, 186 cases of cancer of the large bowel, bladder, and kidney, respectively. As previously described (Augustsson et al, 1999), participation rates were $80 \%$ for controls, and between 68 and $74 \%$ for cases. Reasons for nonparticipation included death, illness, refusal, or loss of questionnaire. An additional 40 respondents (15 controls and 25 cases) had missing dietary data on $>10 \%$ of items and were dropped from the analysis. The sample size for the current study was 538 controls, and 591, 263, 133 cases with cancer of the large bowel, bladder and kidney, respectively. This study was approved by the Ethical Committee of Karolinska Institutet.

\section{Dietary data}

Dietary habits in the 5 years prior to the study were assessed by semiquantitative food frequency questionnaire of 188 food items. Respondents could choose from 10 intake frequency categories ranging from two to three times per day to never.

In addition, more than 100 food samples were analysed to determine acrylamide levels at the NFA. The majority of the foods found to contain high levels of acrylamide were covered by the food frequency questionnaire including French fries, potato crisps, fried potatoes, crisp bread, breakfast cereals, and biscuits. A summary of median acrylamide levels is given in Table 1 . The survey found that foodstuffs that were not fried, deep fried, or oven-baked during production or preparation did not contain any appreciable levels of acrylamide.

A summary measure of acrylamide dose was determined by ranking the following food items on acrylamide dose: potato crisps, French fries, fried potatoes, fried pancakes, pizza, meatballs, breaded fish, cereals, crisp and soft bread, and biscuits. The foodstuffs were ranked according to the median acrylamide content determined by the Swedish NFA as follows: food stuffs without appreciable acrylamide were assigned a rank of 0 ; food stuffs with a median acrylamide content less than $100 \mu \mathrm{g} \mathrm{kg}^{-1}$ were assigned a rank of 1 ; those with a median acrylamide dose $100-$ $200 \mu \mathrm{g} \mathrm{kg}^{-1}$ were assigned a rank of 2 ; those with a median acrylamide dose $200-600 \mu \mathrm{g} \mathrm{kg}^{-1}$ were assigned a rank of 4 ; and those with a median acrylamide dose greater than $600 \mu \mathrm{g} \mathrm{kg}^{-1}$ were assigned a rank of 8 . We converted an individual's intake of each food item to grams per day, multiplied this by the acrylamide level ranking for the specific food item, and summed up the ranks across all of the above foods items for each individual.

Table I Acrylamide concentration for several food groups: Swedish National Food Administration, 2002

\begin{tabular}{lccc}
\hline & $\begin{array}{c}\text { Acrylamide } \\
\text { concentration }\left(\mu \mathbf{~ k g}^{-\mathbf{I}}\right)\end{array}$ & \\
\cline { 2 - 3 } & Median & Min-max & $\begin{array}{c}\text { Number of } \\
\text { samples }\end{array}$ \\
\cline { 2 - 3 } Food group & 1200 & $330-2300$ & 14 \\
\hline Potato crisps & 450 & $300-1100$ & 9 \\
French fries & 300 & & 1 \\
Pan-fried potatoes & 410 & $<30-650$ & 14 \\
Biscuits and crackers & 140 & $<30-1900$ & 21 \\
Crisp breads & 160 & $<30-1400$ & 15 \\
Breakfast cereals & 150 & $120-180$ & 3 \\
Corn chips & 50 & $<30-160$ & 20 \\
Soft breads & 40 & $<30-60$ & 9 \\
Various fried foods (pizza, pancakes, & & & \\
waffles, fish fingers, meatballs, & & & \\
chickenbits, deep fried fish, vegetarian & & & \\
schnitzel, and cauliflower gratin) & & \\
\hline
\end{tabular}

\section{Statistical analysis}

We assessed the risk of each cancer separately for 14 food items found to contain high acrylamide levels (median $>40 \mu \mathrm{g} \mathrm{kg}^{-1}$ ) and for the total summary measure of dietary acrylamide. Unconditional logistic regression was employed to calculate odds ratios (OR), an estimate of the rate ratio, and $95 \%$ confidence intervals (95\% CI).

Quartiles of the summary acrylamide measure were created based on the distribution of the control group, and modelled as categorical variables with the lowest quartile as referent group. Breads were modelled as servings per day. Tests for trend were calculated using likelihood ratio tests, where the categorical medians of each quartile were modelled as ordinal covariates. In multivariate models, we considered as potential confounders smoking (current, former, never), body mass index (categorical), alcohol intake (categorical-colorectal cancer only), fruit and vegetable intake (continuous), saturated fat density (quintiles, categorical), red meat density (quintiles, categorical), and total energy (log transformed). Variables that were statistically significant at $\alpha=0.20$, or which confounded the relation between acrylamide and cancer, were included in the final model (Rothman and Greenland, 1998). Since smoking contributes appreciably to acrylamide exposure, we examined possible differences in cancer risk among smokers and nonsmokers through stratification and through adding interaction term(s) in the models.

Finally, a sensitivity analysis was performed to examine the extent of misclassification by using the median acrylamide concentrations to rank food items and calculate total dietary acrylamide dose. In this approach, we calculated summary measures by reranking individual food items based alternatively on the minimum and maximum acrylamide content measured in the Swedish NFA samples. All statistical analyses were performed using SAS version 8.2.

\section{RESULTS}

Lifestyle and dietary characteristics of cases and controls are shown in Table 2. Weekly intake of French fries and potato crisps by both cases and controls was low, while intake of pan-fried potatoes was more common. About one-fifth of controls consumed three or more slices of crisp bread per day. These data suggest that bread products and pan-fried potatoes account for a major source of dietary acrylamide. Estimated mean daily intake of acrylamide through diet was $27.5 \mu \mathrm{g}$ among controls, based on foodstuffs investigated by the Swedish NFA as of April 2002.

The association between foodstuffs containing high acrylamide levels and risk of cancers of the large bowel and urinary tract is presented in Table 3. Controlling for potential confounders, there is little evidence of an association between any specific baked or fried potato product and cancer risk. While the data suggest a higher risk of bladder cancer for those with daily intake of any fried or baked potatoes (adjusted OR 1.6 (95\% CI 0.7-3.5)), wide CIs preclude definitive assessment. There is no evidence of a positive association between crisp breads and cancer risk. Indeed, the relation between crisp bread and large bowel cancer risk appeared to be inverse ( $P$ for trend $=0.07$ ). No other breads or cereals were related, inversely or positively, with risk of cancer of the large bowel, bladder or kidney. With respect to other fried foods, the data suggest a small increase in risk of large bowel cancer with increasing consumption of breaded fish ( $P$ for trend $=0.11)$. No other fried food items are associated with risk of the studied cancers.

The relative risk of cancer comparing quartiles of daily dietary acrylamide intake based on ranking of food items is presented in Table 4. For colorectal cancer, the risk of cancer decreases with increasing quartiles of acrylamide through diet ( $P$ for tren$\mathrm{d}=0.01$ ). After adjusting for potential confounders, the risk of 
Table 2 Distribution of demographic, lifestyle and dietary factors among controls and cases of large bowel, bladder and kidney cancer

\begin{tabular}{|c|c|c|c|c|}
\hline & $\begin{array}{l}\text { Controls } \\
N(\%)\end{array}$ & $\begin{array}{c}\text { Cancer cases of large bowel } \\
N(\%)\end{array}$ & $\begin{array}{c}\text { Cancer cases of bladder } \\
N(\%)\end{array}$ & $\begin{array}{c}\text { Cancer cases of kidney } \\
\text { N (\%) }\end{array}$ \\
\hline \multicolumn{5}{|l|}{ Characteristics of participants } \\
\hline Males & $272(50.6 \%)$ & $346(58.5 \%)$ & $199(75.7 \%)$ & 71 (53.4\%) \\
\hline Females & $266(49.4 \%)$ & $245(41.5 \%)$ & $64(24.3 \%)$ & $62(46.6 \%)$ \\
\hline \multicolumn{5}{|l|}{ Age group (years) } \\
\hline $51-62$ & |4| (26.2\%) & $167(28.3 \%)$ & $54(20.5 \%)$ & 45 (33.8\%) \\
\hline $73-77$ & $130(24.2 \%)$ & $150(25.4 \%)$ & $68(25.9 \%)$ & $23(17.3 \%)$ \\
\hline Body mass index $\left(\mathrm{kg} \mathrm{m}^{-2}\right)>25.0$ & $205(38.5 \%)$ & $316(53.8 \%)$ & $140(53.5 \%)$ & $70(53.4 \%)$ \\
\hline Current smokers & $130(24.2 \%)$ & $134(22.7 \%)$ & $128(48.7 \%)$ & $34(25.6 \%)$ \\
\hline \multicolumn{5}{|l|}{ Food intake 5 years prior } \\
\hline \multicolumn{5}{|l|}{ Weekly consumption of } \\
\hline Muesli & $172(32.4 \%)$ & $168(28.8 \%)$ & 70 (27.0\%) & $42(31.8 \%)$ \\
\hline Oatmeal & $244(45.3 \%)$ & $211(35.8 \%)$ & $88(33.5 \%)$ & $46(34.6 \%)$ \\
\hline Biscuits & $257(47.8 \%)$ & $302(51.2 \%)$ & $123(47.0 \%)$ & $66(49.6 \%)$ \\
\hline Fried meatballs & $54(10.0 \%)$ & $50(8.5 \%)$ & $27(10.3 \%)$ & $19(14.3 \%)$ \\
\hline Breaded fish & $100(19.5 \%)$ & $132(23.7 \%)$ & $55(22.1 \%)$ & $30(24.0 \%)$ \\
\hline Pancakes/waffles & 181 (33.6\%) & $193(32.7 \%)$ & $80(30.4 \%)$ & $43(32.3 \%)$ \\
\hline \multicolumn{5}{|l|}{$3+$ slices per day of } \\
\hline Crisp bread & $104(21.2 \%)$ & $117(19.8 \%)$ & $57(12.2 \%)$ & $28(31.0 \%)$ \\
\hline Rye bread & $45(8.3 \%)$ & $63(10.6 \%)$ & $35(13.3 \%)$ & $15(11.3 \%)$ \\
\hline White bread & $54(10.0 \%)$ & $61(10.3 \%)$ & $26(9.9 \%)$ & $20(15.0 \%)$ \\
\hline Other dietary factors & Mean (SE) & Mean (SE) & Mean (sE) & Mean (sE) \\
\hline Fruit and vegetables $\left(\mathrm{g} \mathrm{day}^{-1}\right)$ & $275.3(8.2)$ & $250.1(6.8)$ & 234.9 (1 I. I) & 260.6 (15.2) \\
\hline Saturated fat $\left(\mathrm{g} \mathrm{day}^{-1}\right)$ & $35.5(0.7)$ & $38.1(0.7)$ & $40.4(1.3)$ & $36.8(1.5)$ \\
\hline Total calories ( $\mathrm{kcal} \mathrm{day}^{-1}$ ) & $2065.9(27.5)$ & $2186.4(29.3)$ & $2243.0(49.4)$ & $2150.3(62.9)$ \\
\hline Alcohol $\left(\mathrm{g} \mathrm{day}^{-1}\right)$ & $10.8(0.6)$ & $11.8(0.6)$ & $15.2(1.1)$ & $10.8(1.4)$ \\
\hline Acrylamide intake through diet $\left(\mu \mathrm{g} \mathrm{day}^{-1}\right)$ & $27.5(0.6)$ & $28.6(0.6)$ & $29.4(0.9)$ & $28.4(1.2)$ \\
\hline
\end{tabular}

large bowel cancer for those in the highest quartile of dietary acrylamide per day was $40 \%$ lower compared to those consuming in the lowest quartile. The inverse association with large bowel cancer was evident among nonsmokers (adjusted $\mathrm{OR}=0.6$ comparing highest to lowest quartile; $P$ for trend $=0.03$ ) and suggestive among smokers (adjusted $\mathrm{OR}=0.7$ comparing highest to lowest quartile; $P$ for trend $=0.50$ ). The relative risk between dietary acrylamide dose and cancers of the bladder and kidney was essentially null, and was similar for smokers and nonsmokers.

The findings in Table 4 were unaffected by the acrylamide concentration standard used to rank the food items, that is, using the median concentration $v s$ the minimum or maximum concentration. Comparing the highest to lowest quartile, the relative risk of colorectal cancer was 0.6 using the median concentration, 0.6 using the minimum, and 0.7 using the maximum acrylamide concentration based on the NFA data. The relative risk estimates for bladder and kidney cancers were also unaffected by the acrylamide concentration used in rankings (data available on request)

\section{DISCUSSION}

The finding in April 2002 of elevated levels of acrylamide in a variety of foodstuffs were unexpected, and led to a call by public health scientists for additional research on acrylamide (WHO, 2002). Reports from laboratory studies a few months later have provided insight into the biochemical mechanism of acrylamide formation. Acrylamide can be generated during the heating of specific foodstuffs as a result of a Maillard reaction between amino acids and sugars (Mottram et al, 2002; Stadler et al, 2002). In particular, acrylamide formation occurs when the amino acid asparagine, in the presence of sugars, is heated above $100^{\circ} \mathrm{C}$. Potatoes and cereals, which had the highest measured levels of acrylamide in the Swedish NFA survey, are rich in asparagine (Belitz and Grosch, 1999).

This population-based case-control study found no association between dietary exposure to acrylamide in amounts typically ingested by Swedish adults and risk of cancers of the large bowel, bladder, or kidney. For foods with the highest acrylamide levels, namely, potato crisps, French fries, and crisp bread, there was no positive association with cancer risk. Indeed, the risk of cancer of the large bowel decreased with increasing dose of dietary acrylamide. We did observe a slightly higher risk of bladder cancer for those consuming baked or fried potatoes daily compared to never, although the CI includes the null value. Although this positive finding is in line with a previous study (Steineck et al, 1990), our findings overall suggest that components of potatoes other than acrylamide are responsible for this association.

The classification of acrylamide by IARC as a probable human carcinogen (IARC, 1994) was based mainly on in vitro and animal models. Acrylamide induces genetic mutations and chromosomal abnormalities in vitro, and cellular transformation in vivo (IARC, 
Table 3 Consumption of foods with elevated levels of acrylamide, and risk of cancer of the large bowel, bladder and kidney

\begin{tabular}{|c|c|c|c|c|c|c|}
\hline & \multicolumn{2}{|c|}{ Cancer of large bowel } & \multicolumn{2}{|c|}{ Cancer of bladder } & \multicolumn{2}{|c|}{ Cancer of kidney } \\
\hline & $\begin{array}{c}\text { Adjusted }^{\mathrm{a}} \\
\text { OR }(95 \% \mathrm{Cl})\end{array}$ & $\begin{array}{l}\text { Adjusted }^{\mathrm{b}} \\
\text { OR (95\% Cl) }\end{array}$ & $\begin{array}{c}\text { Adjusted }^{\mathrm{a}} \\
\text { OR }(95 \% \mathrm{Cl})\end{array}$ & $\begin{array}{c}\text { Adjusted }^{\mathrm{b}} \\
\text { OR }(95 \% \mathrm{Cl})\end{array}$ & $\begin{array}{c}\text { Adjusted }^{\mathrm{a}} \\
\text { OR (95\% Cl) }\end{array}$ & $\begin{array}{l}\text { Adjusted } \\
\text { OR (95\% C }\end{array}$ \\
\hline \multicolumn{7}{|l|}{ Potatoes } \\
\hline Never & I (Ref) & I (Ref) & I (Ref) & I (Ref) & I (Ref) & I (Ref) \\
\hline Less often & $1.3(1.0-1.7)$ & $1.3(0.9-1.7)$ & $1.1(0.7-1.5)$ & $0.8(0.5-1.3)$ & $0.9(0.6-1.5)$ & $0.9(0.5-1.4)$ \\
\hline 2-3 times per month & $0.9(0.6-1.3)$ & $0.8(0.6-1.2)$ & $0.8(0.5-1.4)$ & $1.0(0.6-1.6)$ & $0.9(0.5-1.6)$ & $0.7(0.4-1.4)$ \\
\hline Weekly & $0.9(0.6-1.5)$ & $0.8(0.5-1.4)$ & $0.7(0.4-1.3)$ & $0.7(0.4-1.1)$ & $0.9(0.4-1.8)$ & $0.7(0.3-1.6)$ \\
\hline \multicolumn{7}{|l|}{ Potato crisps (high levels) } \\
\hline Never & I (Ref) & I (Ref) & I (Ref) & I (Ref) & I (Ref) & I (Ref) \\
\hline Less often & $1.3(1.0-1.7)$ & $1.3(1.0-1.7)$ & $1.2(0.9-1.7)$ & $1.2(0.8-1.7)$ & $1.6(1.1-2.4)$ & $1.7(1.1-2.6)$ \\
\hline 2-3 times per month & $0.9(0.6-1.4)$ & $0.8(0.5-1.3)$ & $1.2(0.7-2.1)$ & $1.3(0.7-2.3)$ & $0.9(0.4-1.9)$ & $0.9(0.4-1.9)$ \\
\hline Weekly & $1.4(0.8-2.2)$ & $1.3(0.8-2.1)$ & $0.9(0.5-1.8)$ & $0.9(0.4-1.8)$ & $0.8(0.3-2.0)$ & $0.7(0.3-1.9)$ \\
\hline \multicolumn{7}{|c|}{ Pan-Fried Potatoes (high levels) } \\
\hline Never & I (Ref) & I (Ref) & I (Ref) & I (Ref) & I (Ref) & I (Ref) \\
\hline Less often & $1.0(0.7-1.6)$ & $1.0(0.6-1.5)$ & $1.1(0.6-2.0)$ & $1.1(0.5-2.1)$ & $0.8(0.4-1.6)$ & $0.7(0.4-1.5)$ \\
\hline 2-3 times per month & $1.0(0.6-1.6)$ & $0.9(0.5-1.4)$ & $1.2(0.6-2.3)$ & $1.2(0.6-2.3)$ & $0.9(0.5-1.8)$ & $0.8(0.4-1.7)$ \\
\hline Weekly & $1.0(0.6-1.5)$ & $0.8(0.5-1.2)$ & $1.4(0.8-2.5)$ & $1.3(0.7-2.4)$ & $0.8(0.4-1.6)$ & $0.7(0.4-1.4)$ \\
\hline \multicolumn{7}{|c|}{ Potatoes au Gratin (unknown levels) } \\
\hline Never & I (Ref) & I (Ref) & I (Ref) & I (Ref) & I (Ref) & I (Ref) \\
\hline Less often & $1.1(0.7-1.7)$ & $1.2(0.8-1.8)$ & $0.7(0.4-1.1)$ & $0.7(0.4-1.1)$ & $0.8(0.4-1.5)$ & $0.8(0.4-1.5)$ \\
\hline 2-3 times per month & $1.1(0.7-1.7)$ & $1.2(0.7-1.9)$ & $0.7(0.4-1.2)$ & $0.7(0.4-1.3)$ & $0.8(0.4-1.5)$ & $0.8(0.4-1.7)$ \\
\hline \multirow{2}{*}{\multicolumn{7}{|c|}{ Total potatoes fried or baked }} \\
\hline & & & & & & \\
\hline Less often/Never & I (Ref) & I (Ref) & I (Ref) & I (Ref) & I (Ref) & I (Ref) \\
\hline 2-3 per month & $1.0(0.7-1.4)$ & $0.9(0.6-1.2)$ & $1.2(0.7-1.9)$ & $1.2(0.7-2.0)$ & $1.1(0.6-1.8)$ & $1.0(0.6-1.8)$ \\
\hline Weekly & $0.8(0.6-1.1)$ & $0.7(0.5-1.0)$ & $1.2(0.8-1.9)$ & $1.1(0.7-1.8)$ & $0.9(0.5-1.4)$ & $0.7(0.4-1.3)$ \\
\hline Daily & $1.6(0.9-2.7)$ & $1.2(0.6-2.2)$ & $1.7(0.8-3.4)$ & $1.6(0.7-3.5)$ & I.I $(0.4-2.7)$ & $0.8(0.3-2.2)$ \\
\hline
\end{tabular}

Bread

Crisp bread (moderate levels)

None

I-2 slices per day

3 slices per day

$4+$ slices per day

Rye bread (moderate levels)

None

I-2 slices per day

3 slices per day

$4+$ slices per day

White bread (moderate levels)

None

1-2 slices per day

3 slices per day

$4+$ slices per day

Cereal

Comflakes (moderate levels)

Never

Less often

2-3 times per month

I-2 per week

$3+$ per week

Muesli (moderate levels)

Never

Less often

2-3 times per month

1-2 per week

$3+$ per week

I (Ref)

I.0 (0.7-1.4)

$0.9(0.6-1.4)$

$0.8(0.5-1.3)$

I (Ref)

$1.0(0.8-1.3)$

$1.6(0.9-3.0)$

$0.9(0.5-1.6)$

I (Ref)

$1.3(1.0-1.7)$

$1.5(0.8-2.7)$

$0.9(0.6-1.6)$

I (Ref)

I.5 (I.I-2.0)

$1.0(0.7-1.6)$

$1.3(0.9-1.9)$

$1.3(0.9-1.9)$

I (Ref)

$0.9(0.7-1.2)$

$1.3(0.7-2.6)$

$0.9(0.6-1.4)$

I.0 (0.7-1.4)

I (Ref)

I.0 (0.7-1.4)

$0.8(0.5-1.3)$

$0.7(0.4-1.1)$

I (Ref)

$0.9(0.7-1.2)$

$1.5(0.8-2.8)$

$0.7(0.4-1.3)$

I (Ref)

$1.2(0.9-1.6)$

$1.3(0.7-2.3)$

$0.7(0.4-1.3)$

I (Ref)

$0.9(0.6-1.3)$

$0.7(0.4-1.2)$

$0.9(0.5-1.6)$

I (Ref)

$0.8(0.5-1.0)$

$1.3(0.6-2.7)$

$1.0(0.6-2.0)$

I (Ref)

$1.2(0.9-1.7)$

$0.9(0.4-2.0)$

$0.9(0.5-1.8)$

I (Ref)

I.5 (I.I-2.0)

$0.9(0.6-1.5)$

$1.3(0.9-1.9)$

$1.2(0.8-1.9)$

I (Ref)

$1.0(0.7-1.4)$

$1.3(0.7-2.7)$

$1.0(0.7-1.6)$

I.I (0.7-1.6)

I (Ref)

$1.0(0.7-1.4)$

$1.0(0.6-1.7)$

$0.9(0.6-1.5)$

I.I (0.6-1.8)

I (Ref)

$0.6(0.4-0.9)$

$0.9(0.3-2.4)$

$0.9(0.6-1.5)$

$0.8(0.5-1.3)$

I (Ref)

$0.8(0.5-1.2)$

$0.6(0.3-1.2)$

$0.7(0.4-1.4)$

I (Ref)

$0.7(0.5-1.0)$

$1.4(0.6-3.0)$

$0.9(0.5-1.8)$

I (Ref)

$1.0(0.7-1.5)$

$0.7(0.3-1.8)$

$0.6(0.3-1.3)$

I (Ref)

I.0 (0.6-1.7)

$0.7(0.7-1.6)$

$1.3(0.6-2.6)$

I (Ref)

I.0 (0.7-1.5)

$1.7(0.7-4.3)$

$1.3(0.6-3.0)$

I (Ref)

$0.9(0.6-1.3)$

$1.8(0.8-4.3)$

$1.3(0.6-2.7)$

I (Ref)

$0.9(0.6-1.3)$

$0.9(0.5-1.7)$

$0.8(0.5-1.3)$

$1.0(0.6-1.8)$

I (Ref)

$0.7(0.5-1.2)$

$0.9(0.3-2.8)$

I.I $(0.6-1.8)$

$1.0(0.6-1.7)$

I (Ref)

$1.0(0.6-1.7)$

$1.4(0.7-2.6)$

I.I $(0.6-2.0)$

$0.8(0.4-1.6)$

I (Ref)

$0.8(0.4-1.3)$

$1.2(0.4-3.5)$

I.I (0.6-1.9)

$1.0(0.6-1.7)$

I (Ref)

$0.9(0.6-1.6)$

$0.6(0.3-1.5)$

$1.2(0.6-2.5)$

I (Ref)

$0.9(0.6-1.4)$

$1.7(0.7-4.5)$

$1.2(0.5-2.9)$

I (Ref)

$0.8(0.5-1.3)$

$1.7(0.7-4.1)$

I.0 (0.5-2.3)

I (Ref)

$0.9(0.6-1.6)$

$1.3(0.7-2.6)$

$1.0(0.6-1.9)$

$0.8(0.4-1.6)$

I (Ref)

$0.8(0.5-1.3)$

$1.2(0.4-3.7)$

$1.2(0.6-2.2)$

I.I (0.6-2.0)

Various fried foods

Biscuits (high levels)

Less often/never

2-3 per month

Weekly

Daily

Meatballs (moderate levels)

Never

Less often

2-3 times per month

Weekly

I (Ref)

$1.2(0.9-1.6)$

I.I $(0.8-1.6)$

I.I $(0.7-1.6)$

I (Ref)

$0.6(0.3-1.3)$

$0.7(0.3-1.5)$

$0.6(0.2-1.3)$
I (Ref)

I.I $(0.8-1.5)$

$1.0(0.7-1.4)$

$0.9(0.6-1.4)$

I (Ref)

$0.5(0.2-1.2)$

$0.6(0.3-1.3)$

$0.4(0.2-1.1)$
I (Ref)

$0.9(0.6-1.3)$

$1.2(0.7-1.8)$

$0.9(0.5-1.4)$

I (Ref)

$1.7(0.5-8.3)$

$2.3(0.5-10.9)$

$2.0(0.4-10.2)$
I (Ref)

$1.0(0.6-1.5)$

$1.3(0.8-2.1)$

$0.9(0.5-1.7)$

I (Ref)

$0.7(0.1-3.9)$

$0.9(0.2-4.8)$

$0.8(0.1-4.8)$
I (Ref)

I.0 $(0.6-1.6)$

$0.9(0.5-1.6)$

$1.5(0.8-2.6)$

I (Ref)

$0.5(0.2-1.7)$

$0.6(0.2-2.0)$

$0.9(0.3-3.2)$
I (Ref)

I.0 $(0.6-1.6)$

$0.9(0.5-1.7)$

$1.4(0.7-2.6)$

I (Ref)

$0.4(0.1-1.4)$

$0.4(0.1-1.4)$

$0.5(0.2-2.0)$ 
Table 3 (continued)

\begin{tabular}{|c|c|c|c|c|c|c|}
\hline & \multicolumn{2}{|c|}{ Cancer of large bowel } & \multicolumn{2}{|c|}{ Cancer of bladder } & \multicolumn{2}{|c|}{ Cancer of kidney } \\
\hline & $\begin{array}{c}\text { Adjusted }^{a} \\
\text { OR }(95 \% \mathrm{Cl})\end{array}$ & $\begin{array}{c}\text { Adjusted }^{\mathbf{b}} \\
\text { OR }(95 \% \mathrm{Cl})\end{array}$ & $\begin{array}{c}\text { Adjusted }^{a} \\
\text { OR }(95 \% \mathrm{Cl})\end{array}$ & $\begin{array}{c}\text { Adjusted }^{\mathbf{b}} \\
\text { OR }(95 \% \mathrm{Cl})\end{array}$ & $\begin{array}{c}\text { Adjusted }^{\mathrm{a}} \\
\text { OR (95\% CI) }\end{array}$ & $\begin{array}{l}\text { Adjusted }^{\mathrm{b}} \\
\text { OR }(95 \% \mathrm{CI})\end{array}$ \\
\hline \multicolumn{7}{|c|}{ Breaded fish (moderate levels) } \\
\hline Never & *** & *** & *** & **⿻丷木大 & $* *$ & *** \\
\hline Less often & I (Ref) & I (Ref) & I (Ref) & I (Ref) & I (Ref) & I (Ref) \\
\hline 2-3 times per month & $1.2(0.9-1.6)$ & $1.2(0.9-1.5)$ & $1.4(0.9-2.0)$ & $1.4(0.9-2.1)$ & $0.9(0.6-1.5)$ & $0.9(0.6-1.4)$ \\
\hline Weekly & $1.4(1.0-2.0)$ & $1.3(0.9-1.9)$ & $1.4(0.9-2.1)$ & $1.2(0.7-2.0)$ & $1.3(0.7-2.1)$ & I.I (0.6-2.0) \\
\hline \multicolumn{7}{|c|}{ Pancakes/waffles (moderate levels) } \\
\hline Never & I (Ref) & I (Ref) & I (Ref) & I (Ref) & I (Ref) & I (Ref) \\
\hline Less often & $1.6(0.9-3.0)$ & $1.7(0.9-3.4)$ & $1.0(0.5-2.1)$ & $1.2(0.6-2.7)$ & $2.0(0.6-6.1)$ & $2.6(0.7-9.4)$ \\
\hline 2-3 times per month & $1.4(0.7-2.5)$ & $1.3(0.7-2.5)$ & $0.8(0.4-1.5)$ & $0.9(0.4-1.9)$ & $1.5(0.5-4.5)$ & $1.7(0.5-6.1)$ \\
\hline Weekly & $1.3(0.7-2.5)$ & $1.2(0.6-2.3)$ & $0.7(0.4-1.4)$ & $0.7(0.3-1.6)$ & $1.5(0.5-4.6)$ & $1.6(0.4-5.9)$ \\
\hline
\end{tabular}

${ }^{a}$ Adjusted for matching factors age and gender. ${ }^{b}$ Also adjusted for smoking (current, former, never), BMl (categorical), alcohol intake (categorical-colorectal cancer only), fruit and vegetable intake (continuous), saturated fat density (quintiles, categorical), red meat density (quintiles, categorical), and total energy (log transformed). $*$ * No cases or controls never consumed breaded fish products.

Table 4 Quartiles of daily dietary acrylamide dose based on ranking of food items and risk of cancer of the large bowel, bladder and kidney

\begin{tabular}{|c|c|c|c|c|c|c|}
\hline $\begin{array}{l}\text { Quartiles of ranked } \\
\text { daily acrylamide intake }\end{array}$ & $\begin{array}{l}\text { Adjusted }^{\mathrm{a}} \\
\text { OR (95\% Cl) }\end{array}$ & $\begin{array}{l}\text { Adjusted }^{b} \\
\text { OR (95\% Cl) }\end{array}$ & $\begin{array}{l}\text { Adjusted }^{\mathrm{a}} \\
\text { OR (95\% Cl) }\end{array}$ & $\begin{array}{l}\text { Adjusted }^{\mathbf{b}} \\
\text { OR (95\% Cl) }\end{array}$ & $\begin{array}{l}\text { Adjusted }^{\mathrm{a}} \\
\text { OR (95\% Cl) }\end{array}$ & $\begin{array}{l}\text { Adjusted }^{\mathrm{b}} \\
\text { OR }(95 \% \mathrm{Cl})\end{array}$ \\
\hline \multicolumn{7}{|l|}{ Overall } \\
\hline Quartile I & I (Ref) & I (Ref) & I (Ref) & I (Ref) & I (Ref) & I (Ref) \\
\hline Quartile 2 & $1.0(0.74-1.45)$ & $0.9(0.6-1.3)$ & $1.3(0.8-2.1)$ & $1.1(0.7-1.8)$ & $1.0(0.6-1.8)$ & $1.0(0.6-1.9)$ \\
\hline$P$-value for trend & 0.80 & 0.01 & 0.87 & 0.26 & 0.88 & 0.64 \\
\hline \multicolumn{7}{|l|}{ Nonsmokers } \\
\hline Quartile I & I (Ref) & I (Ref) & I (Ref) & I (Ref) & I (Ref) & I (Ref) \\
\hline Quartile 2 & $1.0(0.7-1.5)$ & $0.9(0.6-1.3)$ & $1.4(0.8-2.5)$ & $1.0(0.6-2.0)$ & $1.0(0.5-1.9)$ & $1.0(0.5-2.0)$ \\
\hline Quartile 3 & $0.9(0.6-1.3)$ & $0.6(0.4-0.9)$ & $0.8(0.5-1.6)$ & $0.6(0.3-1.2)$ & $1.1(0.6-2.1)$ & $0.9(0.4-1.9)$ \\
\hline Quartile 4 & $1.1(0.8-1.7)$ & $0.6(0.3-1.0)$ & $1.2(0.6-2.2)$ & $0.7(0.3-1.6)$ & $1.0(0.5-1.9)$ & $0.7(0.3-1.7)$ \\
\hline Quartile 3 & $0.9(0.4-1.9)$ & $0.9(0.4-2.1)$ & $1.3(0.6-2.8)$ & I.I (0.5-2.5) & $1.8(0.5-5.9)$ & $1.5(0.4-6.2)$ \\
\hline Quartile 4 & $0.7(0.4-1.5)$ & $0.7(0.3-2.1)$ & $1.0(0.5-2.2)$ & $1.0(0.4-2.3)$ & $1.2(0.4-4.1)$ & $1.3(0.2-6.3)$ \\
\hline$P$-value for trend & 0.31 & 0.51 & 0.99 & 0.88 & 0.74 & 0.72 \\
\hline
\end{tabular}

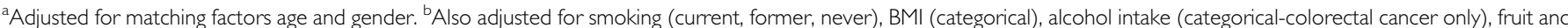
vegetable intake (continuous), saturated fat density (quintiles, categorical), red meat density (quintiles, categorical), and total energy (log transformed).

1994). Long-term studies in rats and mice supported a doseexposure relation between acrylamide and risk of cancer of the lung, mammary gland, thyroid, oral cavity, and intestinal and reproductive tract (Bull et al, 1984; Johnson et al, 1986). Moreover, animals administered acrylamide orally (Paulsson et al, 2002) or fed a diet high in fried foods (Tareke et al, 2000) had higher levels of haemoglobin DNA adducts compared to unexposed animals.

The human data were less clear and limited to occupational settings. In a small cohort of 371 workers exposed to acrylamide through organic dyes, cancer mortality was higher than expected, mainly because of deaths from cancer of the digestive tract and respiratory system (Sobel et al, 1986). More recently, Marsh et al (1999) in a cohort of 8500 workers with potential occupational exposure found little evidence for an excess risk of cancer mortality overall. While an excess of thyroid cancer and a doseexposure relation with pancreatic cancer were suggested, the wide
CIs precluded definitive assessments. The study had greater power to detect an effect of lung cancer, which showed minimal excess risk between both workers exposed and unexposed to acrylamide compared to the general population. Among 200 construction workers exposed to high levels of acrylamide for 20 months, $80 \%$ had haemoglobin adduct levels above the normal background range (Hagmar et al, 2001).

One interpretation of our null finding is that no association between dietary acrylamide and cancer risk exists, implying that species differences negate extrapolating from experimental animals to humans, as shown for other carcinogens (IARC, 1987). In addition, human intake of dietary acrylamide is several folds lower than doses tested in animal experiments. Acrylamide intake within the range of human exposure may thus be effectively detoxified. Although a comprehensive coverage of dietary acrylamide was not possible, certain aspects of our study would support the validity of 
its negative findings. It was large and population-based with reasonably high response rates, thus reducing the possibility of selection bias while the data on demographic, lifestyle, and dietary, covariates would have reduced the opportunity for confounding in the analysis.

Could certain limitations and possible biases have attenuated a true positive association? First, the acrylamide content of a number of food items has not yet been characterised, so our values of daily intake of dietary acrylamide may be underestimated. Measurement errors of acrylamide intake in cases and controls would entail such attenuation (Rothman and Greenland, 1998). There was also variability in acrylamide dose across brands of a given food. It is relevant that it is ranking of individuals with respect to exposure, rather than absolute intake, that determines the calculated relative risk in case-control studies. In fact, when comparing an abbreviated $v s$ extensive food frequency questionnaire, increasing the number of food items improves the ranking, and thereby the relative risk, only to a small degree (Voskuil et al, 1999). In our own data, we found the relative risk estimates comparing quartiles of total acrylamide dose to be insensitive to the concentration of acrylamide used to rank individual food items. Although we possibly capture only partial intake of acrylamide, it is likely that we have a ranking that is valid for drawing conclusions.

Second, a true association may be concealed if the level of exposure in the studied population is low and/or if the range of variation is limited. Of the food items found to contain the highest levels of acrylamide, only biscuits and pan-fried potatoes were commonly consumed by this population. Risk assessment models for humans suggest that lifetime risk for cancer is $0.7-4.5$ per 1000 based on consumption of $1 \mu \mathrm{g}$ acrylamide per $\mathrm{kg}$ body weight per day (US EPA, 1985; WHO, 1985). In our study, less than $2 \%$ of the population was estimated to intake acrylamide through diet at these levels. Acrylamide intake through dietary sources may thus be effectively detoxified within the range of human exposure. This hypothesis is suggested for heterocyclic amines, which cause cancer when given to rodents, but does not appear to in humans,

\section{REFERENCES}

Augustsson K, Skog K, Jagerstad M, Dickman PW, Steineck G (1999) Dietary heterocyclic amines and cancer of the colon, rectum, bladder, and kidney: a population-based study. Lancet 353: $703-707$

Belitz HD, Grosch W (1999) Food Chemistry. New York: Springer

Bull RJ, Robinson M, Laurie RD, Stoner GD, Greisiger E, Meier JR et al (1984) Carcinogenic effects of acrylamide in Sencar and A/J mice. Cancer Res 44: $107-111$

Hagmar L, Tornqvist M, Nordander C, Rosen I, Bruze M, Kautiainen A et al (2001) Health effects of occupational exposure to acrylamide using haemoglobin adducts as biomarkers of internal dose. Scand J Work Environ Health 27: 219-226

IARC (1987) Overall Evaluations of Carcinogenecity: An updating of IARC Monographs, Vols. 1-42, Supplement No. 7. Lyon: International Agency for Research on Cancer

IARC (1994) Monographs on the Evaluation of Carcinogen Risk to Humans: Some Industrial Chemicals, No. 60, Lyon: International Agency for Research on Cancer

Johnson KA, Gorzinski SJ, Bodner KM et al (1986) Chronic toxicity and oncogenicity study on acrylamide incorporated in the drinking water of Fischer 344 rats. Toxicol Appl Pharmacol 85: 154-168

Marsh GM, Lucas LJ, Youk AO, Schall LC (1999) Mortality patterns among workers exposed to acrylamide: 1994 follow up. Occup Environ Med 56: $181-190$

Mottram DS, Wedzicha BL, Dodson AT (2002) Food chemistry: acrylamide is formed in the Maillard reaction. Nature 419: 448-449

Paulsson B, Grawe J, Tornqvist M (2002) Haemoglobin adducts and micronucleus frequencies in mouse and rat after acrylamide or $\mathrm{N}$ methylolacrylamide treatment. Mutat Res 516: $101-111$ where doses are typically millionth of those given in studies of animal carcinogenicity (Augustsson et al, 1999).

Third, residual confounding is a possible explanation for some of our findings, such as the inverse association between total acrylamide dose and large bowel cancer. The food items under study contain a multitude of nutrients. Although we have controlled analytically for total energy, saturated fat, meat, and fruits and vegetables, it may be difficult to disentangle the protective effect of specific nutrients from that of acrylamide. Lastly, although no excess risk was observed for the three major cancers studied, we cannot rule out a possible excess risk of other cancer sites. However, large bowel, bladder, and kidney would be likely target sites, because acrylamide and its metabolite glycidamide are detoxified by glutathione conjugation, are water soluble, and are absorbed quickly in the digestive tract and excreted via the urine (IARC, 1994).

This first study of dietary acrylamide in relation to three major human cancers is reassuring. Needless to say, additional epidemiological evidence is required, notably for other cancer sites as well as for neurological and other disorders. While the null hypothesis of no effect can never be scientifically proven, it would be useful to determine cooking methods that avoid acrylamide formation during food preparation. Since measuring error in acrylamide intake would entail underestimation of any true association with cancer risk, validation studies of acrylamide dose using existing food questionnaires should be a high priority.

\section{ACKNOWLEDGEMENTS}

The present study was made possible by STINT, by supporting collaboration between Karolinska Institutet and Harvard University. The original case-control study was supported by the Swedish Cancer Society. We thank Dr Walt Willett for his invaluable advice concerning the analysis of dietary data and acrylamide.
Rothman KJ, Greenland S (1998) Modern Epidemiology, 2nd ed. Philadelphia: Lippincott-Raven Publishers

Sobel W, Bond GG, Parsons TW, Brenner FE (1986) Acrylamide cohort mortality study. Br J Ind Med 43: 785-788

Stadler RH, Blank I, Varga N, Robert F, Hau J, Guy PA, Robert MC, Riediker S (2002) Food chemistry: Acrylamide from Maillard reaction products. Nature 419: 449-450

Steineck G, Hagman U, Gerhardsson M, Norell SE (1990) Vitamin A supplements, fried foods, fat and urothelial cancer. A case-reference study in Stockholm in 1985-87. Int J Cancer 45: 1006-1011

Swedish National Food Administration. Information about acrylamide in food Uppsala, Sweden. April 2002. Website: http://192.71.90.8/engakrylanalysresultat.htm

Tareke E, Rydberg P, Karlsson P, Eriksson S, Tornqvist M (2000) Acrylamide: a cooking carcinogen? Chem Res Toxicol 13: $517-522$

US Environmental Protection Agency (1985) Assessment of Health Risks from Exposure to Acrylamide. Washington DC: Office of Toxic Substances

Voskuil DW, Augustsson K, Dickman PW, van't Veer P, Steineck G (1999) Assessing the human intake of heterocyclic amines: limited loss of information using reduced sets of questions. Cancer Epidemiol Biomarkers Prev 8: 809-814

World Health Organization (1985) Acrylamide. Environmental Health Criteria 49. Geneva: WHO

World Health Organization (2002) Additional research on acrylamide in food essential, scientists declare. Geneva, June 2002. Website: http:// www.who.int/inf/en/pr-2002-51.html 